\title{
CHAMPAGNE SUBREGIONS OF THE UNIT BALL WITH UNAVOIDABLE BUBBLES
}

\author{
Stephen J. Gardiner and Marius Ghergu \\ University College Dublin, School of Mathematical Sciences \\ Belfield, Dublin 4, Ireland; stephen.gardiner@ucd.ie \\ University College Dublin, School of Mathematical Sciences \\ Belfield, Dublin 4, Ireland; marius.ghergu@ucd.ie
}

\begin{abstract}
This paper is concerned with the type of region that arises when infinitely many disjoint closed balls, or "bubbles", are removed from the unit ball of Euclidean space. It characterises those configurations of balls which carry full harmonic measure for the resultant region.
\end{abstract}

\section{Main results}

Let $B(x, r)$ denote the open ball of centre $x$ and radius $r$ in Euclidean space $\mathbf{R}^{n}$ $(n \geq 2)$, and let $B=B(0,1)$. This paper is concerned with domains of the form $\Omega=B \backslash\left(\cup_{k} \bar{B}\left(x_{k}, r_{k}\right)\right)$, where the closed balls $\bar{B}\left(x_{k}, r_{k}\right)$ are pairwise disjoint, $\left|x_{k}\right| \rightarrow 1$ and $\sup _{k} r_{k} /\left(1-\left|x_{k}\right|\right)<1$. Such domains are known as champagne regions and the removed balls are referred to collectively as the bubbles. It is convenient to assume that $0 \in \Omega$. The main problem is to determine those configurations of bubbles which cause the unit sphere to carry no harmonic measure for $\Omega$. Since this is equivalent to the bubbles being unavoidable for Brownian motion starting at 0 , we will describe such configurations as unavoidable.

When $n=2$ Akeroyd [3] has shown that, for any $\varepsilon>0$, there are champagne regions for which $\cup_{k} \bar{B}\left(x_{k}, r_{k}\right)$ is unavoidable and yet $\sum_{k} r_{k}<\varepsilon$. Ortega-Cerdà and Seip [7], also working in the disc, subsequently showed that this phenomenon can occur for any given sequence $\left(x_{k}\right)$ satisfying

$$
\inf _{j \neq k} \frac{\left|x_{j}-x_{k}\right|}{1-\left|x_{k}\right|}>0
$$

and

$$
B(x, a(1-|x|)) \cap\left\{x_{k}: k \in \mathbf{N}\right\} \neq \emptyset \quad(x \in B)
$$

for some $a \in(0,1)$. In this case, if $r_{k}=\left(1-\left|x_{k}\right|\right) \phi\left(\left|x_{k}\right|\right)$ for some decreasing function $\phi:[0,1) \rightarrow(0,1)$, it was shown that the bubbles are unavoidable if and only if

$$
\int_{0}^{1} \frac{1}{(1-t) \log (1 / \phi(t))} d t=\infty .
$$

doi:10.5186/aasfm.2010.3520

2000 Mathematics Subject Classification: Primary 31B20.

Key words: Harmonic measure, Newtonian capacity.

This research was supported by Science Foundation Ireland under Grant 09/RFP/MTH2147, and is also part of the programme of the ESF Network "Harmonic and Complex Analysis and Applications" (HCAA). 
This result was recently extended by O'Donovan [6] to higher dimensions, where the corresponding condition on $\phi$ is

$$
\int_{0}^{1} \frac{\{\phi(t)\}^{n-2}}{1-t} d t=\infty
$$

The main purpose of this paper is to obtain results of this nature for more general champagne subregions of the unit ball, where the separation condition (1) is substantially relaxed. From now on we will assume that $n \geq 3$. Normalised surface area measure on $\partial B$ will be denoted by $\sigma$.

Theorem 1. Let $\Omega$ be a champagne subregion of the unit ball.

(a) If the bubbles are unavoidable, then

$$
\sum_{k} \frac{\left(1-\left|x_{k}\right|\right)^{2}}{\left|y-x_{k}\right|^{n}} r_{k}^{n-2}=\infty \quad \text { for } \sigma \text {-almost every } y \in \partial B \text {. }
$$

(b) Conversely, if (3) holds, together with the separation condition

$$
\inf _{j \neq k} \frac{\left|x_{j}-x_{k}\right|}{r_{k}^{1-2 / n}\left(1-\left|x_{k}\right|\right)^{2 / n}}>0,
$$

then the bubbles are unavoidable.

We note that condition (4) is strictly weaker than (1) when $n \geq 3$. To see that it cannot be omitted, let $K_{j}$ denote the closed cube of centre $\left(1-2^{-j}, 0, \ldots, 0\right)$ and sidelength $2^{-j-1} / \sqrt{n}$, with sides parallel to the coordinate hyperplanes. If, for each $j \in \mathbf{N}$, we choose $2^{j n^{2}}$ disjoint closed balls of radius $2^{-j-3-j n} / \sqrt{n}$ inside $K_{j}$, the resultant configuration of balls is certainly avoidable and yet satisfies (3).

As we will indicate briefly at the end of the paper, our approach to proving Theorem 1 also leads to an improvement of related results for unavoidable configurations of balls in space that have recently been obtained by Carroll and Ortega-Cerdà [5].

Next, following Ortega-Cerdà and Seip [7] and O'Donovan [6], we consider what more can be said when $r_{k}$ is of the form $\left(1-\left|x_{k}\right|\right) \phi\left(\left|x_{k}\right|\right)$, where $\phi:[0,1) \rightarrow(0,1)$ is decreasing. We note that (1) and (2) together imply that the number of points

$$
N_{a}(x)=\#\left[B(x, a(1-|x|)) \cap\left\{x_{k}: k \in \mathbf{N}\right\}\right]
$$

satisfies $1 \leq N_{a}(x) \leq b$ for some constants $a \in(0,1)$ and $b>1$. In the next result we will allow $N_{a}(x)$ to grow, as $|x| \rightarrow 1$, like some increasing function $M:[0,1) \rightarrow[1, \infty)$ where

$$
M(1-t / 2) \leq c M(1-t) \quad(0<t \leq 1),
$$

for some $c>1$.

Theorem 2. Let $\phi$ and $M$ be as above, and let $\Omega$ be a champagne subregion of the unit ball, where $r_{k}=\left(1-\left|x_{k}\right|\right) \phi\left(\left|x_{k}\right|\right)$.

(a) If the bubbles are unavoidable and there are constants $a \in(0,1)$ and $b>0$ such that $N_{a}(x) \leq b M(|x|)$ for all $x \in B$, then

$$
\int_{0}^{1} \frac{\{\phi(t)\}^{n-2} M(t)}{1-t} d t=\infty .
$$


(b) Conversely, if (5) holds, together with the separation condition

$$
\inf _{j \neq k} \frac{\left|x_{j}-x_{k}\right|}{\left\{\phi\left(\left|x_{k}\right|\right)\right\}^{1-2 / n}\left(1-\left|x_{k}\right|\right)}>0,
$$

and there are constants $a \in(0,1)$ and $b>0$ such that $N_{a}(x) \geq b M(|x|)$ for all $x \in B$, then the bubbles are unavoidable.

We can now deduce a higher dimensional version of Akeroyd's result.

Corollary 3. Let $\varepsilon>0$.

(a) There is a champagne subregion of the unit ball satisfying (1), (2) and $\sum_{k} r_{k}^{n-1}<\varepsilon$, such that the bubbles are unavoidable.

(b) For any $\alpha>n-2$ there is a champagne subregion of the unit ball such that $\sum_{k} r_{k}^{\alpha}<\varepsilon$ and the bubbles are unavoidable.

To see that part (b) of the corollary is sharp, suppose we have a champagne subregion of the unit ball such that $\sum_{k} r_{k}^{n-2}<\infty$. By omitting finitely many of the balls we can arrange that $\bar{B}\left(x_{k}, r_{k}\right) \subset B \backslash B(0,1 / 2)$ for all $k$ and $\sum_{k} r_{k}^{n-2}<2^{-n}$. By subadditivity this would imply that the Newtonian capacity of the union of the remaining balls is at most $2^{-n}$, whence the associated capacitary potential is valued at most $1 / 4$ at 0 . Thus these balls are avoidable, and it follows that the full collection of balls is also avoidable.

The above results will be proved using Whitney decompositions, two different types of quasiadditivity of Newtonian capacity, and minimal thinness. For potential theoretic background material we refer to the book [4].

\section{Proof of Theorem 1}

Let $E=\cup_{k} \bar{B}\left(x_{k}, r_{k}\right)$, so that $\Omega=B \backslash E$. For a positive superharmonic function $u$ on $B$ we define the usual reduced function

$$
R_{u}^{E}=\inf \{v: v \text { is positive and superharmonic on } B \text { and } v \geq u \text { on } E\} .
$$

Then $E$ is unavoidable if and only if $R_{1}^{E}(0)=1$. The Poisson kernel for $B$ with pole at $y \in \partial B$ is given by

$$
P(x, y)=\frac{1-|x|^{2}}{|x-y|^{n}} \quad(x \in B) .
$$

Since $\int P(\cdot, y) d \sigma(y) \equiv 1$, we see that

$$
R_{1}^{E}(0)=R_{\int P(\cdot, y) d \sigma(y)}^{E}(0)=\int_{\partial B} R_{P(\cdot, y)}^{E}(0) d \sigma(y) .
$$

Hence $E$ is unavoidable if and only if $R_{P(\cdot, y)}^{E}(0)=1=P(0, y)$ for $\sigma$-almost every $y \in \partial B$. By the connectedness of $\Omega$ and the maximum principle,

$$
E \text { is unavoidable if and only if } R_{P(\cdot, y)}^{E} \equiv P(\cdot, y) \text { for } \sigma \text {-a.e. } y \in \partial B \text {. }
$$

We note, for use below, that the condition $R_{P(\cdot, y)}^{A} \not \equiv P(\cdot, y)$ characterizes minimal thinness with respect to $B$ of a set $A \subset B$ at a boundary point $y$ (see Chapter 9 of [4]). 
Next we choose a Whitney decomposition of $B$; this is a collection of closed cubes $\left\{Q_{m}: m \in \mathbf{N}\right\}$ with sides parallel to the coordinate hyperplanes such that their union is $B$, their interiors are pairwise disjoint, and

$$
\operatorname{diam}\left(Q_{m}\right) \leq \operatorname{dist}\left(Q_{m}, \partial B\right) \leq 4 \operatorname{diam}\left(Q_{m}\right) \quad(m \in \mathbf{N})
$$

(see Chapter VI of Stein [8]). A Wiener-type criterion for minimal thinness (see Corollary 7.4.4 of Aikawa and Essén [2]), based on the quasiadditivity of Green capacity with respect to Whitney decompositions, tells us that

$$
R_{P(\cdot, y)}^{E} \equiv P(\cdot, y) \text { if and only if } \sum_{m} \frac{\left\{\operatorname{dist}\left(Q_{m}, \partial B\right)\right\}^{2}}{\left\{\operatorname{dist}\left(y, Q_{m}\right)\right\}^{n}} \mathscr{C}\left(E \cap Q_{m}\right)=\infty,
$$

where $\mathscr{C}(\cdot)$ denotes Newtonian capacity.

We will need the following elementary lemma, the proof of which is left to the reader. It relies on the fact that $\sup _{k} r_{k} /\left(1-\left|x_{k}\right|\right)<1$, and the constant $c_{1}$ below depends on the value of this supremum.

Lemma 4. There is a constant $c_{1}>1$ such that, for any $Q_{m}$ and any $\bar{B}\left(x_{k}, r_{k}\right)$ which intersects $Q_{m}$ :

$$
\frac{1}{c_{1}} \leq \frac{\operatorname{dist}\left(Q_{m}, \partial B\right)}{1-\left|x_{k}\right|} \leq c_{1}, \quad \text { and } \quad \frac{1}{c_{1}} \leq \frac{\operatorname{dist}\left(y, Q_{m}\right)}{\left|y-x_{k}\right|} \leq c_{1} \quad \text { for all } y \in \partial B .
$$

Now suppose that $E$ is unavoidable. By (7) and (9),

$$
\sum_{m} \frac{\left\{\operatorname{dist}\left(Q_{m}, \partial B\right)\right\}^{2}}{\left\{\operatorname{dist}\left(y, Q_{m}\right)\right\}^{n}} \mathscr{C}\left(E \cap Q_{m}\right)=\infty \text { for } \sigma \text {-almost every } y \in \partial B \text {. }
$$

By the countable subadditivity of Newtonian capacity,

$$
\mathscr{C}\left(E \cap Q_{m}\right)=\mathscr{C}\left(\left[\cup_{k} \bar{B}\left(x_{k}, r_{k}\right)\right] \cap Q_{m}\right) \leq \sum_{k} \mathscr{C}\left(\bar{B}\left(x_{k}, r_{k}\right) \cap Q_{m}\right) .
$$

Since the number of cubes $Q_{m}$ which intersect a given ball $\bar{B}\left(x_{k}, r_{k}\right)$ is bounded above by a constant $c_{2}$, independent of $k$, and since

$$
\mathscr{C}\left(\bar{B}\left(x_{k}, r_{k}\right) \cap Q_{m}\right) \leq \mathscr{C}\left(\bar{B}\left(x_{k}, r_{k}\right)\right)=r_{k}^{n-2},
$$

we see from the above lemma that

$$
\begin{aligned}
\sum_{m} \frac{\left\{\operatorname{dist}\left(Q_{m}, \partial B\right)\right\}^{2}}{\left\{\operatorname{dist}\left(y, Q_{m}\right)\right\}^{n}} \mathscr{C}\left(E \cap Q_{m}\right) & \leq \sum_{k} \sum_{m} \frac{\left\{\operatorname{dist}\left(Q_{m}, \partial B\right)\right\}^{2}}{\left\{\operatorname{dist}\left(y, Q_{m}\right)\right\}^{n}} \mathscr{C}\left(\bar{B}\left(x_{k}, r_{k}\right) \cap Q_{m}\right) \\
& \leq c_{1}^{n+2} c_{2} \sum_{k} \frac{\left(1-\left|x_{k}\right|\right)^{2}}{\left|y-x_{k}\right|^{n}} r_{k}^{n-2} .
\end{aligned}
$$

Hence (3) follows from (10). This proves part (a) of Theorem 1.

For part (b) we require the following.

Lemma 5. Suppose that

$$
\frac{\left|x_{j}-x_{k}\right|}{r_{k}^{1-2 / n}\left(1-\left|x_{k}\right|\right)^{2 / n}} \geq 4 c_{1}^{4 / n} \quad(j \neq k),
$$


where $c_{1}$ is as in Lemma 4. Then there is a constant $c_{3}>0$ depending only on $n$ such that, for any Whitney cube $Q_{m}$,

$$
\mathscr{C}\left(E \cap Q_{m}\right) \geq c_{3} \sum_{k} \mathscr{C}\left(\bar{B}\left(x_{k}, r_{k}\right) \cap Q_{m}\right) .
$$

Proof of Lemma. We will establish this by applying a different type of quasiadditivity property of Newtonian capacity to a scaled version of $E \cap Q_{m}$. Let $\lambda_{n}$ denote the Lebesgue measure of $B$. A result of Aikawa and Borichev [1] tells us that, if $F$ is an analytic subset of $\cup_{k} B\left(y_{k}, \rho_{k}\right)$, where $\rho_{k} \leq \lambda_{n}^{-1 / 2} 2^{-n / 2}$ for all $k$, and if the balls $\left\{B\left(y_{k}, \lambda_{n}^{-1 / n} \rho_{k}^{1-2 / n}\right): k \in \mathbf{N}\right\}$ are pairwise disjoint, then

$$
\sum_{k} \mathscr{C}\left(F \cap B\left(y_{k}, \rho_{k}\right)\right) \leq C(n) \mathscr{C}(F)
$$

where $C(n)$ is a constant that depends only on $n$. We will apply it to the set $E^{\circ} \cap Q_{m}$ after scaling by the factor

$$
\alpha=\frac{\lambda_{n}^{-1 / 2} 2^{-n / 2}}{c_{1} \operatorname{dist}\left(Q_{m}, \partial B\right)}
$$

Thus we define

$$
F=\left(\cup_{k} B\left(y_{k}, \rho_{k}\right)\right) \cap \alpha Q_{m}
$$

where $y_{k}=\alpha x_{k}, \rho_{k}=\alpha r_{k}$ and $\alpha Q_{m}=\left\{\alpha x: x \in Q_{m}\right\}$. If $B\left(x_{k}, r_{k}\right) \cap Q_{m} \neq \emptyset$, then we see from Lemma 4 and (11) that

$$
\rho_{k}=\alpha r_{k} \leq \alpha\left(1-\left|x_{k}\right|\right) \leq \alpha c_{1} \operatorname{dist}\left(Q_{m}, \partial B\right)=\lambda_{n}^{-1 / 2} 2^{-n / 2}
$$

and

$$
\begin{aligned}
\frac{\left|y_{j}-y_{k}\right|}{\rho_{k}^{1-2 / n}} & =\frac{\alpha\left|x_{j}-x_{k}\right|}{\alpha^{1-2 / n} r_{k}^{1-2 / n}} \geq 4\left\{\alpha c_{1}^{2}\left(1-\left|x_{k}\right|\right)\right\}^{2 / n} \\
& \geq 4\left\{\alpha c_{1} \operatorname{dist}\left(Q_{m}, \partial B\right)\right\}^{2 / n}=2 \lambda_{n}^{-1 / n} \quad(j \neq k) .
\end{aligned}
$$

Thus the hypotheses of the above quasiadditivity theorem are satisfied. The estimate (12) follows, using the facts that $\mathscr{C}(\alpha A)=\alpha^{n-2} \mathscr{C}(A)$ for any analytic set $A$ and that $\mathscr{C}\left(\bar{B}\left(x_{k}, r_{k}\right) \cap Q_{m}\right)=\mathscr{C}\left(B\left(x_{k}, r_{k}\right) \cap Q_{m}\right)$ for all $m$ and $k$.

Now suppose that (3) and (4) hold. We choose $\delta \in(0,1)$ small enough so that

$$
\frac{\left|x_{j}-x_{k}\right|}{\left(\delta r_{k}\right)^{1-2 / n}\left(1-\left|x_{k}\right|\right)^{2 / n}} \geq 4 c_{1}^{4 / n} \quad(j \neq k)
$$

and define $E_{\delta}=\cup_{k} \bar{B}\left(x_{k}, \delta r_{k}\right)$. From Lemmas 4 and 5 we see that

$$
\sum_{m} \frac{\left\{\operatorname{dist}\left(Q_{m}, \partial B\right)\right\}^{2}}{\left\{\operatorname{dist}\left(y, Q_{m}\right)\right\}^{n}} \mathscr{C}\left(E_{\delta} \cap Q_{m}\right) \geq \frac{c_{3}}{c_{1}^{n+2}} \sum_{k} \sum_{m} \frac{\left(1-\left|x_{k}\right|\right)^{2}}{\left|y-x_{k}\right|^{n}} \mathscr{C}\left(\bar{B}\left(x_{k}, \delta r_{k}\right) \cap Q_{m}\right) .
$$

Subadditivity of capacity implies that, for each $k$, there exists $m$ such that

$$
\mathscr{C}\left(\bar{B}\left(x_{k}, \delta r_{k}\right) \cap Q_{m}\right) \geq c_{2}^{-1} \mathscr{C}\left(\bar{B}\left(x_{k}, \delta r_{k}\right)\right)=c_{2}^{-1} \delta^{n-2} r_{k}^{n-2},
$$

where $c_{2}$ is as above. Thus

$$
\sum_{m} \frac{\left\{\operatorname{dist}\left(Q_{m}, \partial B\right)\right\}^{2}}{\left\{\operatorname{dist}\left(y, Q_{m}\right)\right\}^{n}} \mathscr{C}\left(E_{\delta} \cap Q_{m}\right) \geq \frac{c_{3} \delta^{n-2}}{c_{1}^{n+2} c_{2}} \sum_{k} \frac{\left(1-\left|x_{k}\right|\right)^{2}}{\left|y-x_{k}\right|^{n}} r_{k}^{n-2},
$$


and (3), (7) and (9) together show that $E_{\delta}$, and hence also $E$, is unavoidable. This completes the proof of Theorem 1.

\section{Proof of Theorem 2}

Suppose firstly that $E$ is unavoidable and that $N_{a}(x) \leq b M(|x|)$ for all $x \in B$. Since $r_{k}=\left(1-\left|x_{k}\right|\right) \phi\left(\left|x_{k}\right|\right)$, we see from Theorem 1(a) that

$$
\sum_{k} \frac{\left(1-\left|x_{k}\right|\right)^{n}}{\left|y-x_{k}\right|^{n}}\left\{\phi\left(\left|x_{k}\right|\right)\right\}^{n-2}=\infty \quad \text { for } \sigma \text {-almost every } y \in \partial B \text {. }
$$

Any given centre $x_{k}$ belongs to some Whitney cube $Q_{m}$. Clearly

$$
1-\left|x_{k}\right| \leq \operatorname{dist}\left(Q_{m}, \partial B\right)+\operatorname{diam}\left(Q_{m}\right),
$$

so

$$
1-\left|x_{k}\right| \leq 5 \operatorname{diam}\left(Q_{m}\right) \quad \text { and } \quad 1-\left|x_{k}\right| \leq 2(1-|x|) \quad\left(x \in Q_{m}\right),
$$

by (8). Also, by Lemma 4 ,

$$
\left|y-x_{k}\right| \geq \frac{1}{c_{1}} \operatorname{dist}\left(y, Q_{m}\right) \geq \frac{1}{2 c_{1}}\left\{\operatorname{dist}\left(y, Q_{m}\right)+\operatorname{diam}\left(Q_{m}\right)\right\} \geq \frac{|y-x|}{2 c_{1}} \quad\left(x \in Q_{m}\right),
$$

SO

$$
\frac{\left(1-\left|x_{k}\right|\right)^{n}}{\left|y-x_{k}\right|^{n}}\left\{\phi\left(\left|x_{k}\right|\right)\right\}^{n-2} \leq\left\{10 c_{1} \operatorname{diam}\left(Q_{m}\right)\right\}^{n} \frac{\left\{\phi\left((2|x|-1)^{+}\right)\right\}^{n-2}}{|y-x|^{n}} \quad\left(x \in Q_{m}\right),
$$

in view of the fact that $\phi$ is decreasing. Since the number of centres $x_{k}$ that belong to $Q_{m}$ is bounded above by $C(a, c, n) b M\left((2|x|-1)^{+}\right)$for all $x \in Q_{m}$, we see that

$\sum_{k} \frac{\left(1-\left|x_{k}\right|\right)^{n}}{\left|y-x_{k}\right|^{n}}\left\{\phi\left(\left|x_{k}\right|\right)\right\}^{n-2} \leq C(a, c, n) b c_{1}^{n} \int_{B} \frac{\left\{\phi\left((2|x|-1)^{+}\right)\right\}^{n-2} M\left((2|x|-1)^{+}\right)}{|y-x|^{n}} d x$.

Integration with respect to $d \sigma(y)$, together with (13) and the fact that

$$
\int_{\partial B}|y-x|^{-n} d \sigma(y)=\frac{1}{1-|x|^{2}} \quad(x \in B)
$$

by the harmonicity of the Poisson kernel, yields

$$
\int_{B} \frac{\left\{\phi\left((2|x|-1)^{+}\right)\right\}^{n-2} M\left((2|x|-1)^{+}\right)}{1-|x|^{2}} d x=\infty .
$$

Hence

$$
\int_{1 / 2}^{1} \frac{\{\phi(2 t-1)\}^{n-2} M(2 t-1)}{1-t} d t=\infty
$$

and (5) follows. Thus part (a) of Theorem 2 is established.

To prove part (b), suppose that (5) and (6) hold, and that $N_{a}(x) \geq b M(|x|)$ for all $x \in B$. Let $y \in \partial B$ and define

$$
z_{i}=\left(1-\frac{\alpha^{i}}{2}\right) y \quad(i \in \mathbf{N}), \quad \text { where } \quad \alpha=\frac{1-a}{1+a} .
$$

The balls $\left\{B\left(z_{i}, a\left(1-\left|z_{i}\right|\right)\right)\right\}$ are then pairwise disjoint. Since

$$
1-|x| \geq(1-a) \frac{\alpha^{i}}{2} \text { and }|y-x| \leq(1+a) \frac{\alpha^{i}}{2} \text { when } x \in B\left(z_{i}, a\left(1-\left|z_{i}\right|\right)\right),
$$


we see that

$$
\begin{aligned}
\sum_{k} \frac{\left(1-\left|x_{k}\right|\right)^{2}}{\left|y-x_{k}\right|^{n}} r_{k}^{n-2} & =\sum_{k} \frac{\left(1-\left|x_{k}\right|\right)^{n}}{\left|y-x_{k}\right|^{n}}\left\{\phi\left(\left|x_{k}\right|\right)\right\}^{n-2} \\
& \geq \sum_{i} \sum_{\left\{k: x_{k} \in B\left(z_{i}, a\left(1-\left|z_{i}\right|\right)\right)\right\}} \frac{\left(1-\left|x_{k}\right|\right)^{n}}{\left|y-x_{k}\right|^{n}}\left\{\phi\left(\left|x_{k}\right|\right)\right\}^{n-2} \\
& \geq \alpha^{n} b \sum_{i}\left\{\phi\left(1-(1-a) \frac{\alpha^{i}}{2}\right)\right\}^{n-2} M\left(\left|z_{i}\right|\right) \\
& \geq C(a, b, c, n) \sum_{i}\left\{\phi\left(1-(1-a) \frac{\alpha^{i}}{2}\right)\right\}^{n-2} M\left(1-(1-a) \frac{\alpha^{i+1}}{2}\right) \\
& \geq C(a, b, c, n) \int_{1-(1-a) \alpha / 2}^{1} \frac{\{\phi(t)\}^{n-2} M(t)}{1-t} d t=\infty .
\end{aligned}
$$

Hence $E$ is unavoidable, by Theorem 1(b), using the fact that (6) corresponds to (4) in this case.

\section{Proof of the corollary}

To prove part (a) of the corollary, let $\left\{x_{k}: k \in \mathbf{N}\right\}$ be an enumeration of the centres of the Whitney cubes that do not contain 0 , and define

$$
\phi(t)=\frac{\{1-\log (1-t)\}^{-1 /(n-2)}}{10 \sqrt{n}} .
$$

Then

$$
\int_{0}^{1} \frac{\{\phi(t)\}^{n-2}}{1-t} d t=\infty
$$

and, in view of (14), the balls $\bar{B}\left(x_{k},\left(1-\left|x_{k}\right|\right) \phi\left(\left|x_{k}\right|\right)\right)$ lie inside the corresponding Whitney cubes, and so will be disjoint. Thus we can apply Theorem 2(b), with $M(t) \equiv 1$, to see that $E$ is unavoidable. Further,

$$
\begin{aligned}
\sum_{k} r_{k}^{n-1} & =C(n) \sum_{k} \frac{\left(1-\left|x_{k}\right|\right)^{n-1}}{\left\{1-\log \left(1-\left|x_{k}\right|\right)\right\}^{(n-1) /(n-2)}} \\
& \leq C(n) \int_{B} \frac{1}{(1-|x|)\{1-\log (1-|x|)\}^{(n-1) /(n-2)}} d x<\infty
\end{aligned}
$$

by (14). By omitting a finite number of the balls, we can arrange that $\sum_{k} r_{k}^{n-1}$ is arbitrarily small.

In proving part (b), we may assume that $\alpha \in(n-2, n-1)$. Let $\phi(t)=c_{0}(1-t)^{\beta}$, where $\beta>(n-1-\alpha) /(\alpha-n+2)$ and $c_{0} \in(0,1)$. We divide each Whitney cube $Q_{m}$ that does not contain 0 into $p_{m}^{n}$ subcubes of equal size, where $p_{m}$ is the integer part of $\left\{\operatorname{dist}\left(Q_{m}, \partial B\right)\right\}^{\beta(2-n) / n}$. Let $\left\{x_{k}: k \in \mathbf{N}\right\}$ be an enumeration of the centres of all such subcubes. Then (6) holds. Also, $N_{a}(x) \geq\{\phi(|x|)\}^{2-n}$ for all $x \in B$, for a suitable choice of $a \in(0,1)$, and the balls $\bar{B}\left(x_{k}, r_{k}\right)$, where $r_{k}=\left(1-\left|x_{k}\right|\right) \phi\left(\left|x_{k}\right|\right)$, will be pairwise disjoint, provided we choose $c_{0}$ to be small enough. Since (5) holds 
with $M(t)=\{\phi(t)\}^{2-n}$, Theorem 2(b) shows that $E$ is unavoidable. Further,

$$
\begin{aligned}
\sum_{k} r_{k}^{\alpha} & \leq c_{0}^{\alpha} \sum_{k}\left(1-\left|x_{k}\right|\right)^{(1+\beta) \alpha} \\
& \leq c_{0}^{\alpha} c_{1}^{(1+\beta) \alpha} \sum_{m}\left\{\operatorname{dist}\left(Q_{m}, \partial B\right)\right\}^{(1+\beta) \alpha} p_{m}^{n} \\
& \leq c_{0}^{\alpha} C(n, \alpha, \beta) \int_{B}(1-|x|)^{\beta(\alpha-n+2)+\alpha-n} d x<\infty,
\end{aligned}
$$

by our choice of $\beta$. The result now follows, as before.

\section{Unavoidable configurations of balls in space}

It is also natural to consider domains of the form $\omega=\mathbf{R}^{n} \backslash\left(\cup_{k} \bar{B}\left(x_{k}, r_{k}\right)\right)$, where the balls $\bar{B}\left(x_{k}, r_{k}\right)$ are pairwise disjoint, $0 \in \omega$ and $\left|x_{k}\right| \rightarrow \infty$, and to ask when the balls are unavoidable, that is, when they carry full harmonic measure for $\omega$. This is a simpler problem since the underlying domain is the whole of space, rather than $B$, and the question reduces to asking when the set $F=\cup_{k} \bar{B}\left(x_{k}, r_{k}\right)$ is non-thin at infinity (see Theorem 7.6.5 in [4]). For each $j \in \mathbf{N}$ we form the closed cube of centre 0 and sidelength $3^{j}$ with sides parallel to the coordinate hyperplanes, divide it into $3^{n}$ subcubes of sidelength $3^{j-1}$, and discard the central cube. Let $\left\{\mathscr{R}_{m}\right\}$ be an enumeration of the resulting collection of cubes. Wiener's criterion tells us that $F$ is non-thin at infinity if and only if

$$
\sum_{m}\left\{\operatorname{dist}\left(0, \mathscr{R}_{m}\right)\right\}^{2-n} \mathscr{C}\left(F \cap \mathscr{R}_{m}\right)=\infty
$$

so this divergence condition characterizes when $F$ is unavoidable. Following the approach of Section 2 we arrive at the following analogue of Theorem 1.

Theorem 6. Let $\omega$ be as above.

(a) If $F$ is unavoidable, then

$$
\sum_{k}\left(\frac{r_{k}}{\left|x_{k}\right|}\right)^{n-2}=\infty .
$$

(b) Conversely, if (15) holds, together with the separation condition

$$
\inf _{j \neq k} \frac{\left|x_{j}-x_{k}\right|}{r_{k}^{1-2 / n}\left|x_{k}\right|^{2 / n}}>0,
$$

then $F$ is unavoidable.

Part (a) above corresponds to Proposition 1 of Carroll and Ortega-Cerdà [5], and has a straightforward proof. Part (b) improves Theorem 1 of [5] where, in place of (16), there is the stronger pair of assumptions that

$$
\inf _{j \neq k}\left|x_{j}-x_{k}\right|>0 \quad \text { and } \quad \sup _{k} r_{k}^{n-2}\left|x_{k}\right|^{2}<\infty .
$$




\section{References}

[1] Aiknwa, H., and A. A. Borichev: Quasiadditivity and measure property of capacity and the tangential boundary behavior of harmonic functions. - Trans. Amer. Math. Soc. 348, 1996, 1013-1030.

[2] Aikawa, H., and M. Essén: Potential theory-selected topics. - Lecture Notes in Math. 1633, Springer, Berlin, 1996.

[3] Akeroyd, J. R.: Champagne subregions of the disk whose bubbles carry harmonic measure. Math. Ann. 323, 2002, 267-279.

[4] Armitage, D. H., and S. J. Gardiner: Classical potential theory. - Springer, London, 2001.

[5] Carroll, T., and J. Ortega-Cerdà: Configurations of balls in Euclidean space that Brownian motion cannot avoid. - Ann. Acad. Sci. Fenn. Math. 32, 2007, 223-234.

[6] O'Donovan, J.: Brownian motion in a ball in the presence of spherical obstacles. - Proc. Amer. Math. Soc. 138, 2010, 1711-1720.

[7] Ortega-Cerdà, J., and K. Seip: Harmonic measure and uniform densities. - Indiana Univ. Math. J. 53, 2004, 905-923.

[8] Stein, E. M.: Singular integrals and differentiability properties of functions. - Princeton Univ. Press, Princeton, N.J., 1970.

Received 14 September 2009 